\title{
OUTLOOK: THE NEXT TWENTY YEARS
}

\author{
H. MURAYAMA \\ Department of Physics, University of California, Berkeley, CA 94720 \\ and \\ Theoretical Physics Groups, Lawrence Berkeley Laboratory, University of California, Berkeley, CA 94720 \\ and \\ School of Natural Sciences, Institute for Advanced Study, Princeton, NJ 08540 \\ E-mail: murayama@ias.edu
}

\begin{abstract}
I present an outlook for the next twenty years in particle physics. I start with the big questions in our field, broken down into four categories: horizontal, vertical, heaven, and hell. Then I discuss how we attack the big questions in each category during the next twenty years. I argue for a synergy between many different approaches taken in our field.
\end{abstract}

\section{Introduction}

I was asked specifically not to give a summary talk of the Symposium, but rather an "outlook" talk. Obviously I will refer to many excellent talks given during this Symposium, but I will not try to cover everything that had been said. If you find that your favorite subject is not covered in my talk, you are welcome to come up here and complain to Keith Ellis.

What I will try to do in this talk is present my own perspective on how our field may develop in the next twenty years. Whenever we talk about the future, a very natural question is whether it is bright, as bright as the illumination of the Chicago skyscrapers we admired at the time of the banquet (Fig. 1), or dark, as dark as parts of the East Coast were this week (Fig. 22). You will see my verdict at the end of the talk. But before getting to the verdict, I'd like to talk about the current situation of our field.

One way to look at our field of particle physics is that it has specialized into so many different subfields. During this Symposium alone, we heard about many exciting subjects and experiments. Here is an incomplete list in a random order: $0 \nu \beta \beta, B$ physics, proton decay, LHC, Higgs, reactor antineutrinos, $K$-physics, Lepton Flavor Violation, Cosmic Microwave Background, string theory, $e^{+} e^{-}$, top quark, accelerator-based neutrinos, lattice QCD, charm physics, Dark Energy, hidden dimensions, neutrino factory, hadron physics, Supersymmetry, Dark Matter, atmospheric neutrinos, Linear Collider, exotics, solar neutrinos, and the increasingly important topics of outreach and politics. People

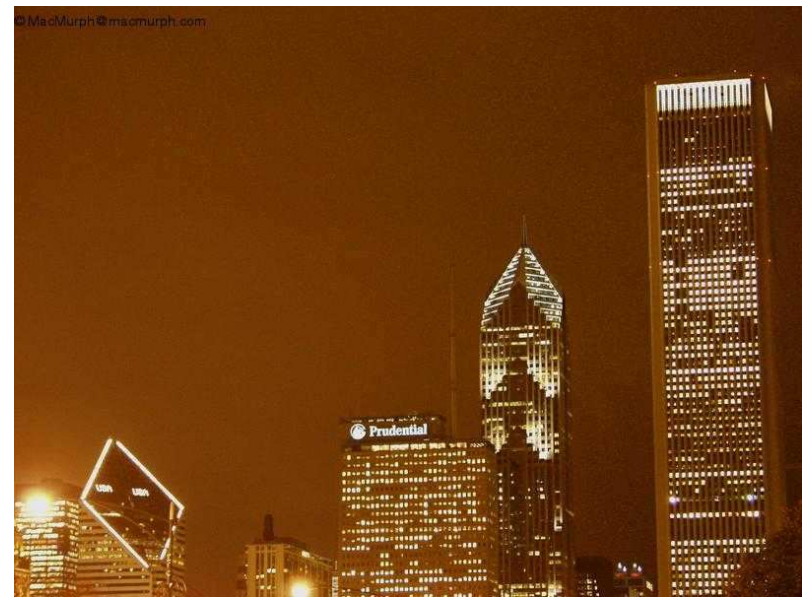

Figure 1. The bright skyline of Chicago.

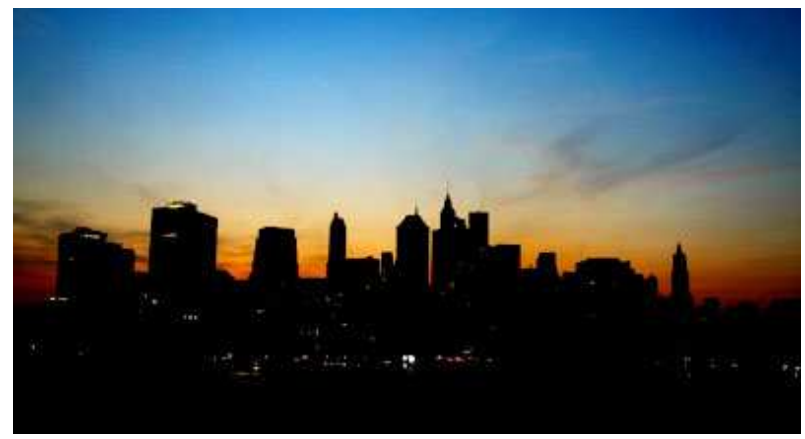

Figure 2. The dark skyline of New York City during the blackout.

may say that the field is completely fragmented. On the other hand, I have a somewhat different view. I think that most of them are heading to a synergy at an important energy scale: TeV. I don't mean that $\mathrm{TeV}$-scale physics will solve all the puzzles we are facing. What I mean is that any of these interest- 


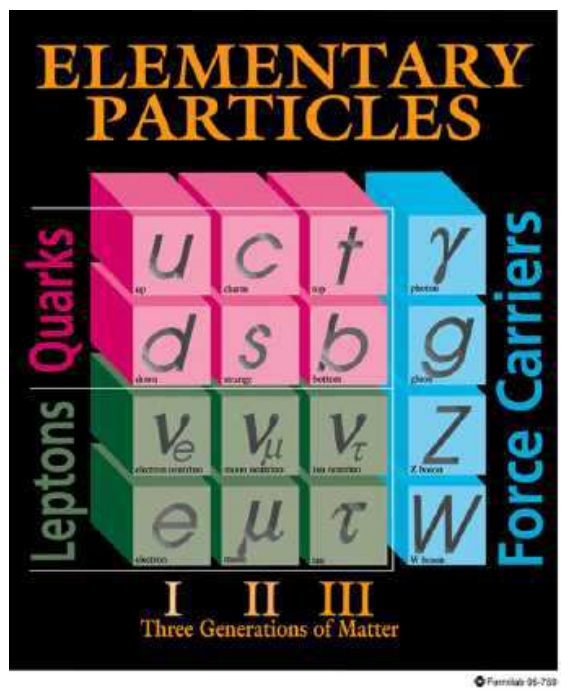

Figure 3. The table of particles in the Standard Model 11 Vertical Questions are concerned with particles in a single generation, while Horizontal Questions refer to the relationship sideways in the table.

fermion masses

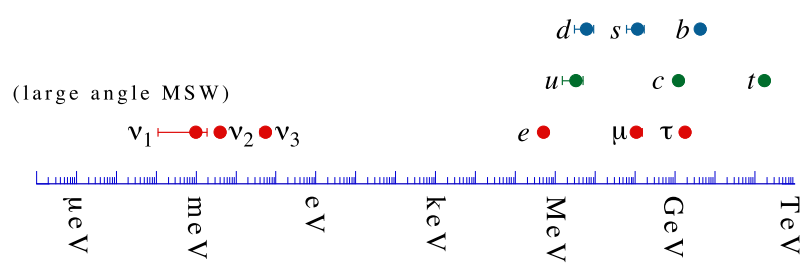

Figure 4. The mass spectrum of quarks and leptons we don't understand.

ing physics topics must once go through the study of the $\mathrm{TeV}$ scale before they reach their own destinations. It is a hub where everybody has to transfer to another flight.

Why do I think so? To see this, let me start enumerating the big questions in our field. I broke them down to four categories.

The first category is what I call the horizontal questions. They are about relationships between the three families of elementary particles (Fig. [3). Why are there three generations and no more? What physics determines their masses and mixings (Fig. 4)? What is the energy scale of that physics? Why do neutrinos have mass and yet they are so light? What is the origin of CP-violation? What is the origin of the matter-antimatter asymmetry in our Universe?

The second category is the vertical questions.

$$
\begin{array}{ll}
Q\left(\mathbf{3}, \mathbf{2},+\frac{1}{6}\right), & u\left(\overline{\mathbf{3}}, \mathbf{1},-\frac{2}{3}\right), \quad d\left(\overline{\mathbf{3}}, \mathbf{1},+\frac{1}{3}\right), \\
L\left(\mathbf{1}, \mathbf{2},-\frac{1}{2}\right), & e(\mathbf{1}, \mathbf{1},+1)
\end{array}
$$

Table 1. The quantum numbers of quarks and leptons we don't understand. Here, all particles are shown in their lefthanded chirality states.

They concern properties within each family of particles (Fig. 3). Why are there three unrelated gauge forces? Why is the strong interaction strong? Why are all electric charges quantized in the same unit? What physics guarantees the seemingly miraculous anomaly cancellation? What physics explains the quantum numbers of quarks and leptons we see (Table 1)? Is there a unified description of all forces? Why is $m_{W} \ll M_{\text {Planck }}$ ? (Hierarchy Problem.)

Recently, we added many questions from the heaven (Fig. [5). What is Dark Matter? What is Dark Energy? Why are we at the special moment when the energy densities of Dark Matter and Dark Energy are the same within a factor of two? ("Why now?" problem.) What exactly was the Big Bang? Why is the Universe so big? (Flatness problem, horizon problem.) How were galaxies and stars (and eventually us) created?

If there are questions from the heaven, there are also questions from the hell. To the best of the collective knowledge of Homo sapiens, we live at the bottom of a strange potential with a wine bottle shape: that's the hell we are in (Fig. 6). Because of this potential, the Bose-Einstein condensate (BEC) of the Higgs boson is supposed to be present in our Universe, and we are swimming in this BEC. What is this Higgs boson thing? Why does it have this strange potential with a negative mass-squared? Why is there only one scalar particle in the Standard Model, designed to do its most mysterious part? Is it elementary or composite? Is it really condensed in our Universe?

We do not have the right to expect that any of these questions can be answered within our lifetime (or ever). Nonetheless there is a good potential for us to answer some or many of them. How exactly do we do it? I will refer to Supersymmetry as an example many times in my talk, but I expect similar stories with any scenario of $\mathrm{TeV}$-scale physics. In any case, $\mathrm{TeV}$ is the key. 


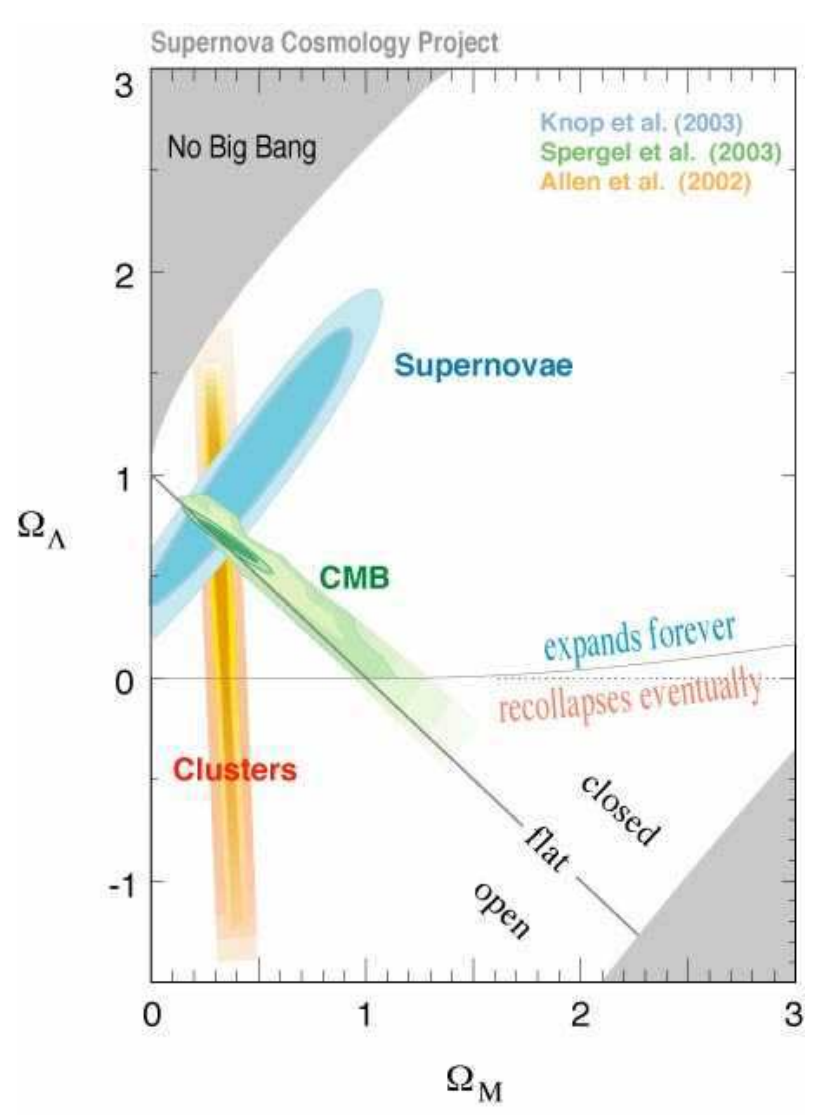

Figure 5. The unknown constituents of the universe.

I have just finished the introduction. Now I move on to discuss each category of questions: hell, heaven, vertical and horizontal. My verdict on the future of our field follows after that.

\section{Hell}

What we know is that the Standard Model of particle physics is completely incapable of answering the big questions I've listed. What we want to do is to look for physics beyond the Standard Model that answers these big questions. By definition, that is physics at shorter distances. In order to talk about the new physics that appears at a some small distance scale, the Standard Model must survive down to whatever that short distance scale is. The problem is that it doesn't. This is the hierarchy problem. It is the main obstacle for us to address the big questions. We can't even get started! (Fig. [7)

To illustrate the reason why we can't even get started, let us rewind the video back to the end of the 19th century. Once upon a time, there was a

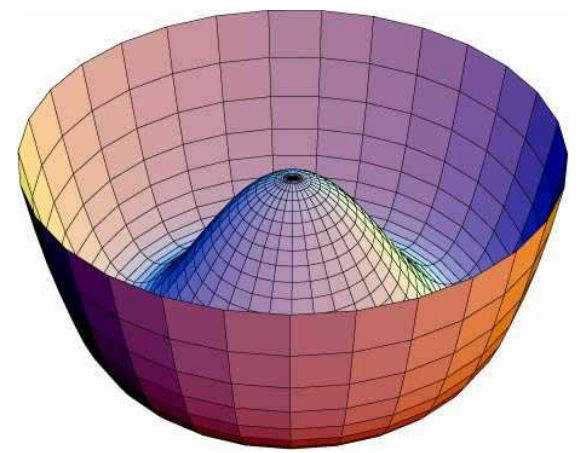

Figure 6. The hell of the Universe we live in and don't know why.

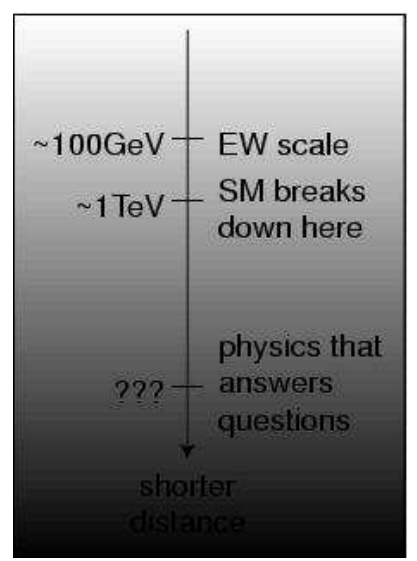

Figure 7. We would like to access physics at a short distance that answers some of the big questions. But before getting there, the Standard Model breaks down around a $\mathrm{TeV}$ and everything at shorter distances is grayed out.

hierarchy problem? It was a crisis about the mass of the electron. We know like charges repel. It is hard to keep electric charge in a small pack because it repels itself. On the other hand, we know the electron is basically point-like. Our best limit is that the "size" of the electron is less than something like $10^{-17} \mathrm{~cm}$. The problem is that, if you want to keep the charge in such a small pack, you need a lot of energy. A naïve guess is that you need at least

$$
\Delta E \sim \frac{\alpha}{r_{e}} \sim 1 \mathrm{GeV} \frac{10^{-17} \mathrm{~cm}}{r_{e}} .
$$

But we know we can't afford it. The energy carried by an electron is just $E=m c^{2}=0.511 \mathrm{MeV}$, nowhere close to what we need. In fact, the best we can do is to pack the charge down to about $10^{-13} \mathrm{~cm}$, which is the so-called classical radius of the electron. In other words, the classical theory of electromagnetism breaks down around this distance scale, and 


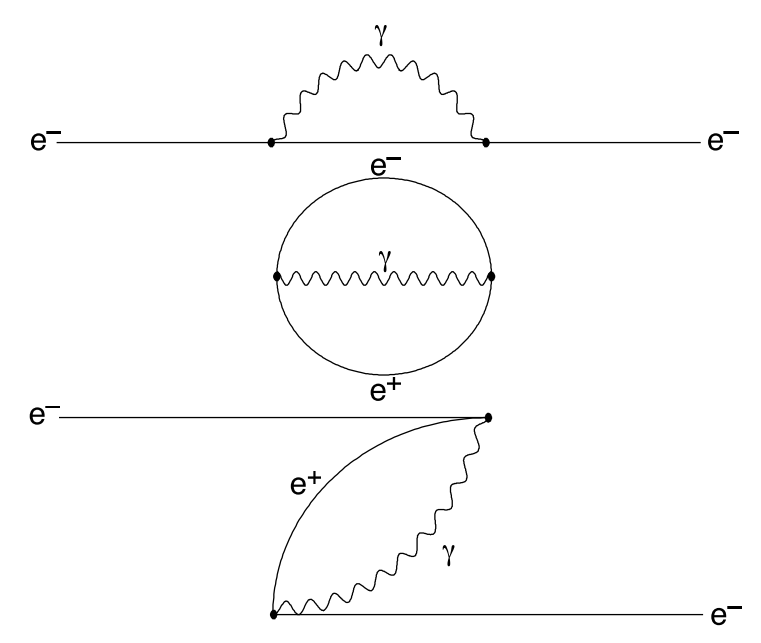

Figure 8. Top: The electron sees the Coulomb field it created itself. Center: The vacuum is full of "bubbles" in which an electron-position pair is created spontaneously and annihilate back to the vacuum within the time allowed by the uncertainty principle. Bottom: An electron may decide to annihilate the positron in the "bubble" while the electron originally in the "bubble" remains as a real particle.

we cannot discuss physics below $10^{-13} \mathrm{~cm}$. We can't get started!

But we don't talk about this problem anymore, because there was a resolution. Antimatter came to the rescue. We solved the crisis by doubling the number of particles. Here is how it works.

The electron creates a Coulomb field around itself, and it feels its own field. Namely, it repels itself (Fig. 8 top). But we discovered antimatter. Moreover, we discovered that the world is quantum mechanical. Once you have these two ingredients, there is an inevitable consequence. The "vacuum" we see isn't empty at all. It constantly creates pairs of electrons and positrons, together with a photon. Of course, energy conservation forbids it, but quantum mechanics allows us to borrow energy as long as nobody notices it. The created pair must annihilate back to the vacuum within the time allowed by the uncertainty principle (Fig. 8 center). Such pairs are called "vacuum bubbles."

When you place an electron in this fluctuating vacuum, it "sees" a positron nearby. Sometimes, it decides to annihilate the positron in the bubble. Then the electron that was originally a part of the bubble now remains as a "real" particle (Fig. [8 bottom). It turns out that this process also contributes to the energy of the electron with a negative sign, that nearly exactly cancels the self-repelling energy

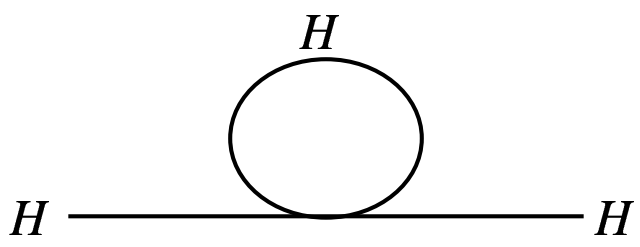

Figure 9. The self-repulsion of the Higgs boson makes it hard to be contained in a small size.

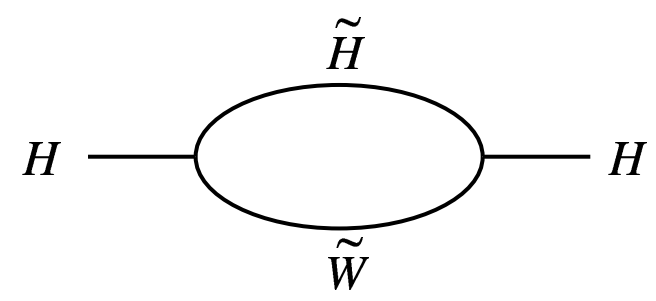

Figure 10. The Supersymmetric attraction diagram cancels the Higgs self-repulsion diagram.

we were worried about. The grand total is roughly

$$
\Delta m_{e} c^{2} \sim m_{e} c^{2} \times \frac{\alpha}{4 \pi} \log \left(m_{e} r_{e}\right)
$$

This is nice. First of all, the additional energy you need is proportional to the original energy (the rest energy $m_{e} c^{2}$ ), and we are talking about a percentage correction. Second, even if you take the smallest size imaginable, namely the Planck size $r_{e} \sim 10^{-33} \mathrm{~cm}$, the size of the correction is only about $10 \%$. Now we can get started to think about physics below $10^{-13} \mathrm{~cm}$.

The problem we are facing now is very similar. The minute you think that we are swimming in the Higgs BEC, you should ask if Higgs can be contained in a small package. It turns out that the Higgs also repels itself because of its self-interaction (Fig. 9). It requires a lot of energy to contain itself. The theory breaks down again, this time around $10^{-17} \mathrm{~cm}$. We are stuck. We can't get started to address the big questions. We can't "see" the interesting physics at shorter distances that answers the big questions.

One way to solve this problem is to assume that history repeats itself. We double the number of particles again. The new particles cancel the contribution from the Higgs self-repelling energy (Fig. 10). This is the idea of Supersymmetry, which makes the Standard Model consistent with whatever physics there is at shorter distances. Indeed, the correction to the Higgs energy is

$$
\Delta m_{H}^{2} \sim \frac{\alpha}{4 \pi} m_{S U S Y}^{2} \log \left(m_{H} r_{H}\right),
$$




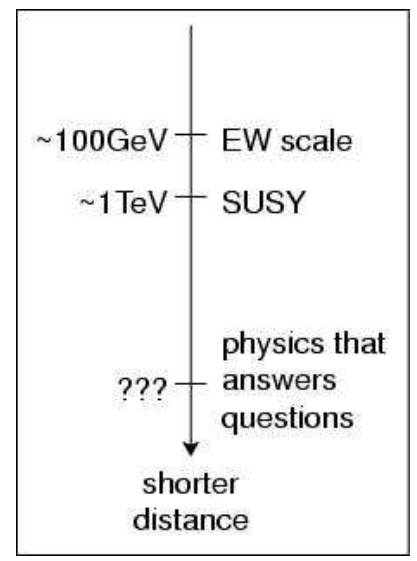

Figure 11. Once the hierarchy problem is solved, we will be allowed to talk about physics at shorter distances to address the big questions.

where $r_{H}$ is the "size" of the Higgs boson. Of course Supersymmetry is not the only solution, but it is true that any solution of this kind appears at the $\mathrm{TeV}$ scale.

Once the hierarchy problem is solved, we can finally get started. It opens the door to the answers to the big questions (Fig. 111). The sky clears up and we can start "seeing" physics at shorter distances. An even more interesting possibility is that the solution itself provides additional probes to physics at shorter distances. We will talk about some examples soon.

In fact, the importance of the $\mathrm{TeV}$-scale has been known since 1933. When Fermi (Fig. 12) wrote down his theory of nuclear beta decay, he knew the relevant energy scale: $G_{F}^{-1 / 2} \simeq 300 \mathrm{GeV}$. It is truly exciting that we are finally getting to this energy scale!

For a long time, theorists, including myself, had been talking about three major directions to solve the hierarchy problem. One is Supersymmetry, which I already talked about. It is the idea that the history repeats itself. Just like antimatter solved the crisis of the electron mass, we double the number of particles. The second direction is to learn from Cooper pairs. The Higgs condensate is a composite made of two fermions. This idea is often called technicolor. These two ideas are two decades old, and many of you have witnessed a nearly religious war between the two camps. The third direction is relatively recent: physics ends at a $\mathrm{TeV}$. The TeVscale is the ultimate scale of physics where quantum gravity manifests itself. It may be superstrings. We may produce blackholes at accelerators or by cosmic

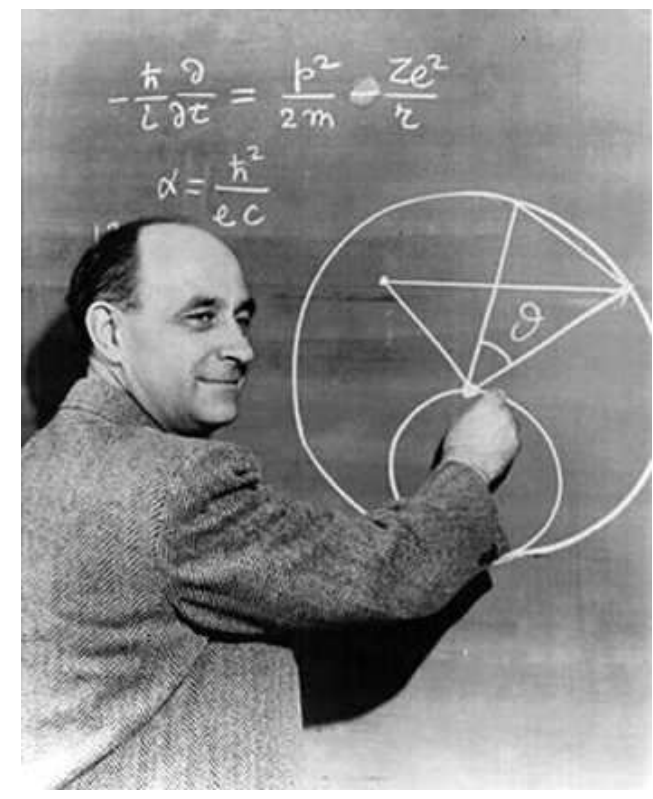

Figure 12. A nice picture of Fermi, except the definition of the fine structure constant is wrong. What was he thinking?

rays. This is possible if there are hidden dimensions curled up in small sizes, somewhere between $10 \mu \mathrm{m}$ to $10^{-17} \mathrm{~cm}$.

But the fact that the third direction was proposed relatively recently suggests that there are many more possibilities we theorists haven't thought of. Indeed, just the last two years have seen an outbreak of new ideas. The Higgs boson may be like pions in QCD, a pseudo-Nambu-Goldstone boson. Models based on this idea are called the "little Higgs" theories. $\frac{3}{3}$ Or maybe the Higgs boson is actually a gauge boson. Extra-dimensional components of a gauge field do not appear to have spins to a four-dimensional observer because they are spinning in extra dimensions. This idea is sometimes called Gauge-Higgs Unification $[4]$ Or maybe there is no Higgs after all, and the reason why $W$ and $Z$ are massive is because they are Kaluza-Klein bosons, running along the extra dimension to acquire their "rest" (for a 4D observer) energies. This idea came to be known as "Higgsless" theories Most recently, I'm pushing the idea of "technicolorful supersymmetry." ${ }^{6}$ You see, I'm pretty ecumenical.

Clearly the landscape of theories is getting more and more complicated (Fig. 13). As the solution to the problem draws near experimentally, theorists are proposing more possibilities. It is increasingly clear 


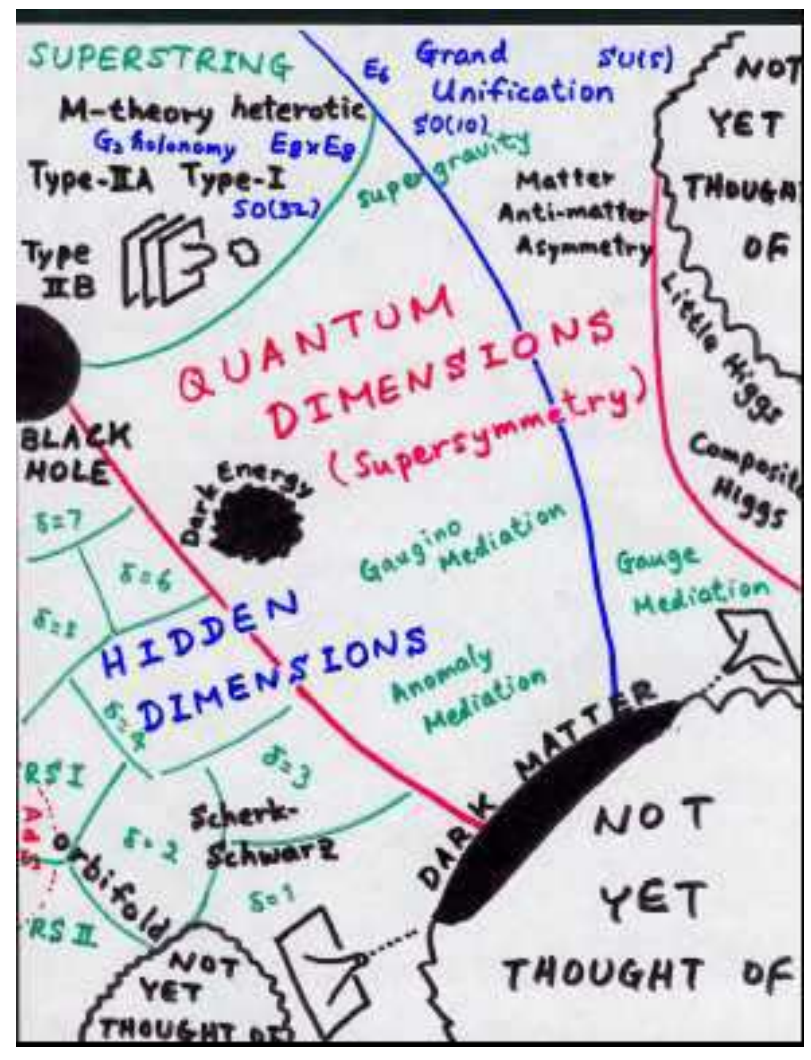

Figure 13. The landscape of theories that has many uncharted territories. The task for experiments is to zoom down to a point on this map.

that all the theoretical possibilities we have talked about, have designed the experiments around, and ran Monte Carlo on, are only a small portion of the land of all theories. There are many islands and continents already labeled on the map, but much of the land is uncharted.

The task for the future experiments is enormous: to zoom in to a point on the map most of which is still uncharted. We need to identify the physics responsible for the Higgs BEC, and it is quite likely that we haven't thought of the right solution yet. We are all excited about the LHC, where we will discover particles and new phenomena that address this issue. Many possibilities will be ruled out. However, new interpretations will necessarily emerge. Then the race will be on. Theorists come up with new interpretations. Experimentalists exclude new interpretations. It will be a long period of elimination. As is always the case, the crucial information is in the details. We would like to elucidate the physics by reconstructing the Lagrangian of the "true theory" term by term from measurements.
In this process, the absolute confidence behind our understanding is crucial, especially when we witness a major discovery. Just for the sake of discussion, let us say that Supersymmetry happens to be the "true theory." It is relatively easy to reach, what I'd like to call, "New York Times-level confidence." We will see a headline like "The Other Half of the World Discovered." But everybody in this auditorium knows that there is a long way to go from this level of confidence to the other level of confidence, which I'd like to call "Halliday-Resnik-level confidence." It will take an incredible level of confidence to put a paragraph like this one in the freshman physics textbook:

We have learned that all particles we observe have unique partners of different spin and statistics, called superpartners, that make our theory of elementary particles valid to small distances.

Upon seeing this slide, one of my colleagues in Berkeley was impressed by the fact that Halliday and Resnik can turn something as exciting as the discovery of Supersymmetry into something this dry and dull. Well, that wasn't my point. My point is that we need to go through many detailed, precise, unambiguous measurements for us to reach this level of confidence.

Again for the sake of discussion, let us say that hidden dimensions happen to be the "true theory." We will see events where the high-energy collisions on our three-dimensional sheet will produce some particles we can see and other particles that disappear into the extra dimensions, such as the graviton (Fig. 14 top). Then we find that the energy and momentum are not balanced apparently. There is clearly something exciting going on. However, such a discovery wouldn't establish the theory. We'd like to know how many of such extra dimensions there are, for instance. One way to address this question is to measure the rates of this kind of events at two different energies at an $e^{+} e^{-}$Linear Collider. The energy dependence of the rates can tell us the number of extra dimensions (Fig. 14 bottom).

Let us pick Supersymmetry again. In this case, the Tevatron and/or LHC will expand our sensitivity in the parameter space greatly beyond where we are, and many precise measurements will be performed at the LHC (Fig. 15). However we will still like to know 


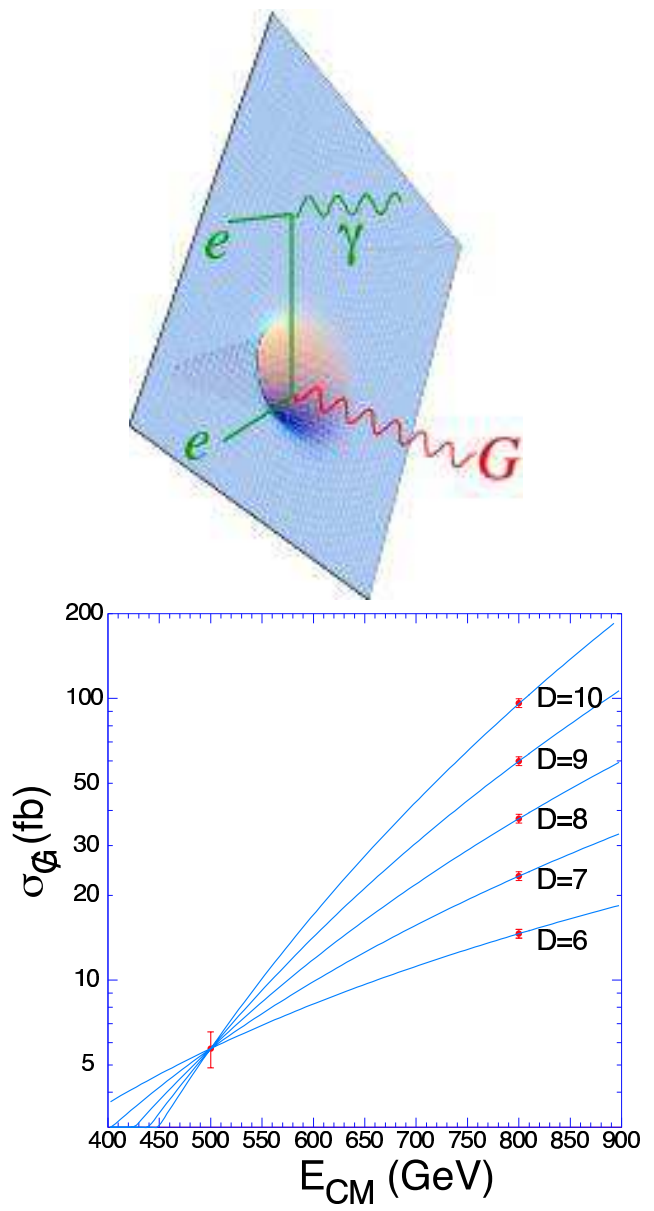

Figure 14. Top: Emission of a graviton into the hidden dimensions. Bottom: The energy dependence of the rates for various number of dimensions?

if the new particles truly have the same quantum numbers as the particle we already know, with their spins differing by $1 / 2$. Again the Linear Collider can determine the quantum numbers and spins, and if they have the correct couplings, etc. (Fig. 16). This way, we will establish Supersymmetry with absolute confidence.

\section{Heaven}

I now turn to the questions from the Heaven. One of the major results this year reported at this Symposium is the study of cosmic microwave background anisotropies by the WMAP satellite. From a global fit, they have reported precise measurements of important cosmological parameters that include 12

$$
h=0.71 \pm 0.04,
$$

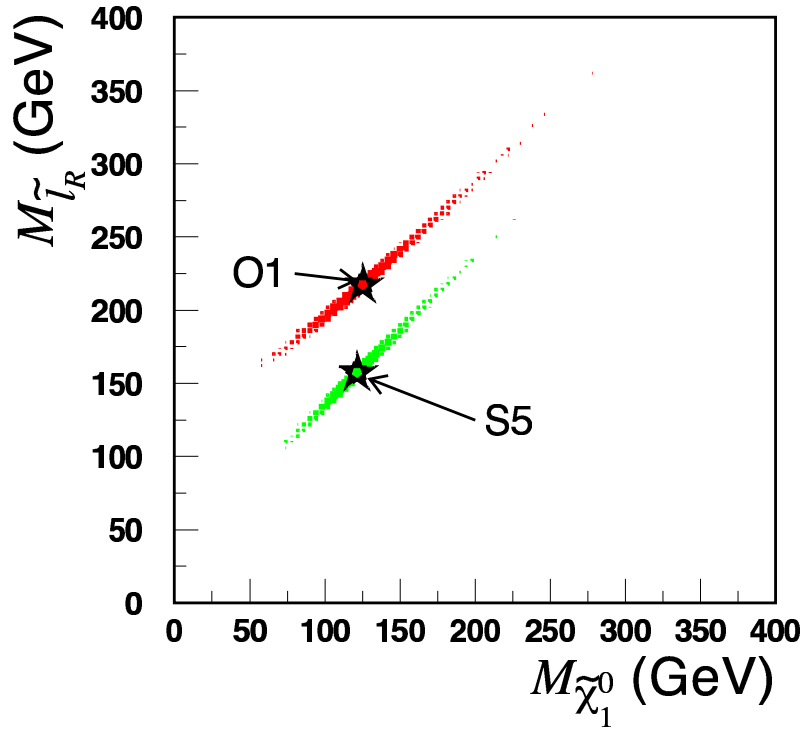

Figure 15. Precision measurement of superparticle masses at the LHC 8

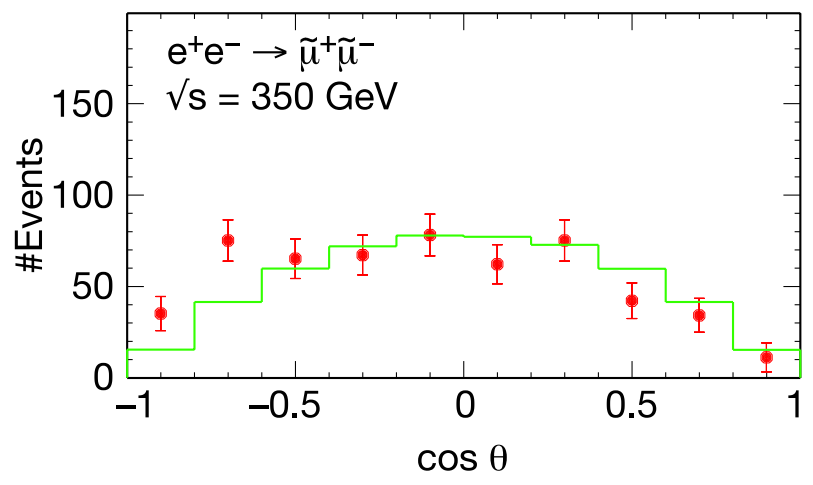

Figure 16. Test if the smuon has spin zero at the LC 9 The spin one case would show an upside down angular distribution.

$$
\begin{aligned}
& \Omega_{M} h^{2}=0.135 \pm 0.009, \\
& \Omega_{b} h^{2}=0.0224 \pm 0.0009, \\
& \Omega_{\text {total }}=1.02 \pm 0.02 .
\end{aligned}
$$

This is yet another big step in precision cosmology. To me, the most important information is that the case for non-baryonic dark matter is now as strong as $12 \sigma:\left|\Omega_{M}-\Omega_{b}\right| h^{2}=0.113 \pm 0.009$.

People have looked for dim stars or big planets that make up Dark Matter in the halo of our galaxy, dubbed MACHOs (Massive Compact Halo Objects). The search resulted in a strong upper limit on the halo fraction of such astronomical objects 13 Instead, we are led to WIMPs (Weakly Interacting Massive Particles). They are stable heavy particles produced in the early Universe when the temperature was as 


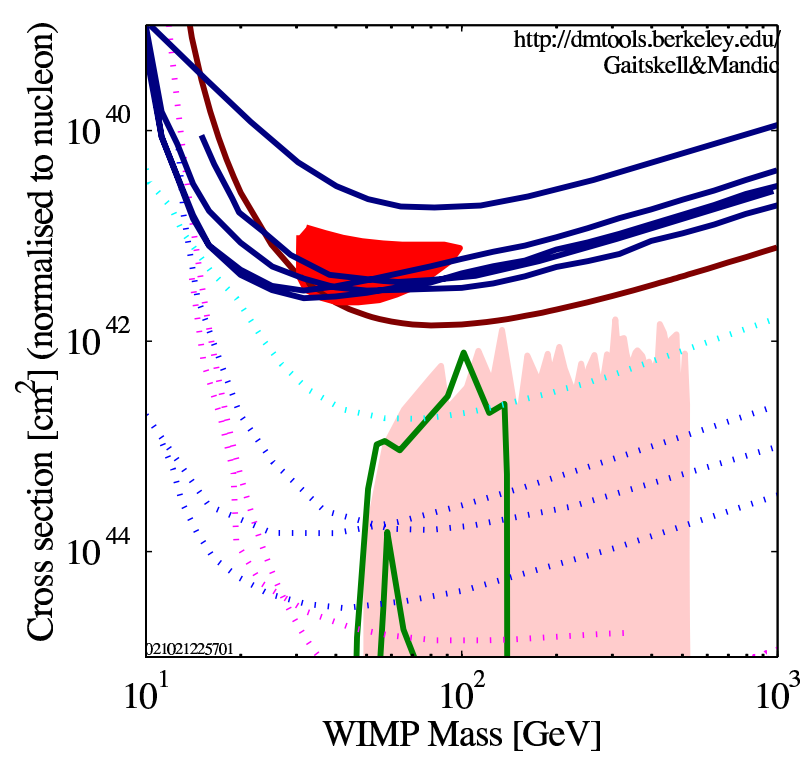

Figure 17. The next-generation Dark Matter search experiments will get into the interesting portion of the WIMP parameter space 10

high as their mass. As the universe cooled, the temperature was so low that they were no longer created. They started to annihilated with each other, but as the universe expands, they saw fewer and fewer of each other and beyond some point they could no longer find each other to annihilate. In this way, they are left-overs from the near complete annihilation. The amount of energy density left over is 11

$$
\Omega_{M}=\frac{0.756(n+1) x_{f}^{n+1}}{g^{1 / 2} \sigma_{a n n} M_{P l}^{3}} \frac{3 s_{0}}{8 \pi H_{0}^{2}} \approx \frac{\alpha^{2} /(\mathrm{TeV})^{2}}{\sigma_{a n n}} .
$$

It is very interesting that weakly coupled (as weak as $\alpha$ ) particles at the $\mathrm{TeV}$ scale can provide the correct energy density to explain the Dark Matter.

A stable, weakly-coupled particle would be an excellent candidate for Dark Matter. Actually, it should be very weakly coupled because ordinary neutrinos would be too strongly coupled and are excluded by the negative search results. There are no such candidate particles in the Standard Model. The candidate most talked about is the Lightest Supersymmetric Particle (LSP), which is the superpartner of the photon or $Z$ in most models. Indeed, the direct search experiments so far have made only a small foray, but the next generation experiments will take a significant bite out of the interesting part of the parameter space (Fig. 17). This way, we will know that Dark Matter is indeed there floating in the halo of our galaxy. On the other hand, we would also

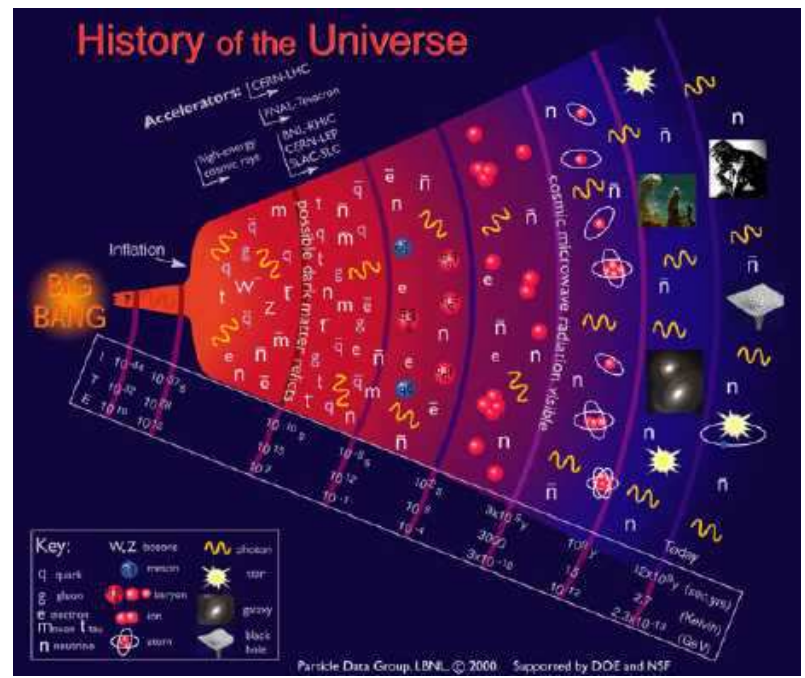

Figure 18. Schematic history of Universe. We have a pretty good grip on physics back to about a second after the Big Bang thanks to CMB and nucleosynthesis. The quark-gluon plasma is physics back at $10^{-5} \mathrm{sec}$. An agreement between accelerator-based data on Dark Matter and cosmological data would provide understanding much closer to The Beginning, back to $10^{-12}$ sec after the Big Bang.

like to know what it is. For this purpose, we'd like to produce ample quantities of Dark Matter in the laboratory to study its properties in detail.

I have argued that the Dark Matter is likely be a $\mathrm{TeV}$-scale electrically neutral weakly interacting particle. There are many such candidates: Lightest Supersymmetric Particle, Lightest Kaluza-Klein particle in universal extra dimension, etc. Given that I expect new particles at the $\mathrm{TeV}$-scale to address the "Hell" problems, it is quite conceivable that one of those particles is stable (or long-lived enough) to be the Dark Matter. If so, it will be accessible at accelerators, such as the LHC and LC. Precision measurements of its mass and couplings to other particles at LHC and LC will allow us to calculate its cosmic abundance. If that calculation based on accelerator experiments turns out to agree with the cosmological observations, it would be a major triumph of modern physics. We will understand the universe all the way back to when it was only about $10^{-12}$ sec old after Big Bang (Fig. [18)!

The Dark Energy is even more mysterious and we should be ready for more surprises. One big question is why we seem to see nearly equal amounts of Dark Energy and Dark Matter now. This is the notorious "Why Now?" problem. We seem to live at a 


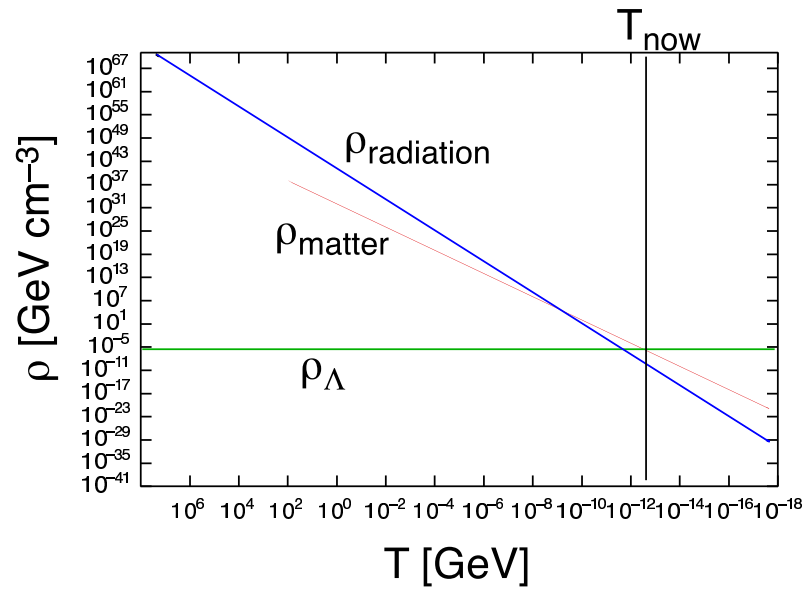

Figure 19. The triple coincidence of three energy densities 14

very special moment in the evolution of universe. It almost feels like we are stepping back from the heliocentric view of Copernicus to the geocentric world of Ptolemy. We physicists all hate the idea that we are special.

Given that the problem is so big, it is useful to step back a little bit and look at the situation globally. Then we find that it is not just Dark Matter and Dark Energy; the "radiation," which basically refers to CMB photons and neutrinos, has a similar energy density as well (Fig. 19). It is actually a triple coincidence problem. We have three lines with different slopes that meet at a single point. Leaving $O(1) \mathrm{nu}-$ merical constants aside, the radiation energy density is $\rho_{\text {rad }} \sim T^{4}$, while the Dark Matter energy density is $\rho_{M} \sim m^{2} T^{3} / M_{P l}$, where $m \sim 1 \mathrm{TeV}$ gives the correct amount as we have seen earlier. In order for the Dark Energy to meet with both of them, we need the Dark Energy density to be $\rho_{\Lambda} \sim\left(\mathrm{TeV}^{2} / M_{P l}\right)^{4}$. Indeed, the observation suggests $\rho_{\Lambda} \approx(2 \mathrm{meV})^{4}$, while $\mathrm{TeV}^{2} / M_{P} l \approx 0.5 \mathrm{meV}$, tantalizingly close. It looks like figuring out $\mathrm{TeV}$-scale physics is crucial for the Dark Energy problem, too.

The parameter we would most like to measure about the Dark Energy is its equation of state. Marc Kamionkowski once told me that the "equation of state" is a misnomer. It is not an equation, but rather a ratio of the pressure to the energy density $w=p / \rho$. Due to some reason, it is called the equation of state, but it is just a number. In any case, the cosmological constant corresponds to $w=-1$, while an evolving dynamical system typically has $w>-1$. A dedicated high-statistics study of high-

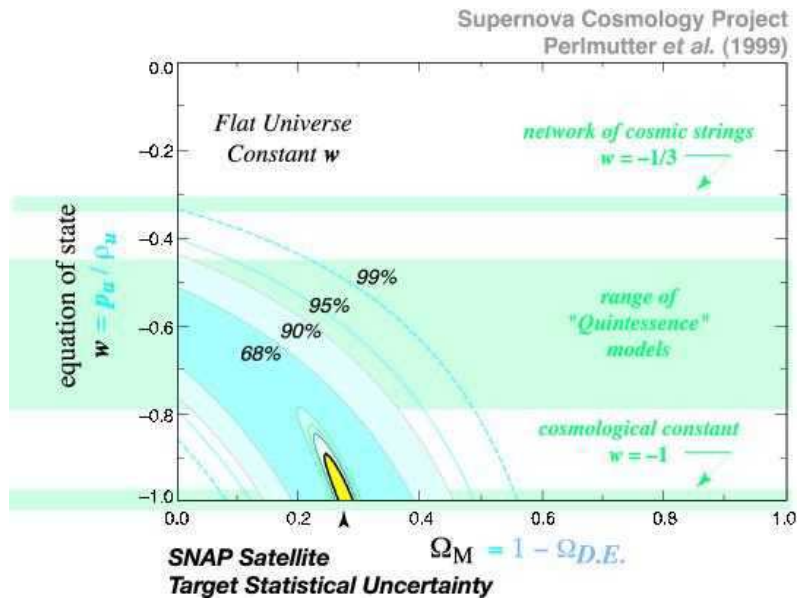

Figure 20. The projective accuracy of the equation of state of the Dark Energy by SNAP 16

redshift supernovae, complemented by the study of nearby ones to pin down the systematic issues would be extremely useful: such as SuperNova Acceleration Probe (SNAP) using a dedicated satellite. It will determine the "equation of state" at a high accuracy. My favorite candidate for Dark Energy, a frustrated network of domain wall $\$ 15$ that leads to $w=-2 / 3$, will be cleanly distinguished from the cosmological constant once SNAP happens (Fig. 20). Once we know the equation of state, we will get the first glimpse of the nature of the Dark Energy. Where to go from there will depend on what we find.

\section{Vertical}

Now on to the Vertical Questions.

Einstein once asked a very simple question. Is there an underlying simplicity behind the vast phenomena in Nature? He dreamed of finding a unified description of all phenomena we see. But he failed to find a unified theory of electromagnetism and his theory of gravity, general relativity.

Indeed, trying to come up with a more universal, more fundamental, more unified theory is in the blood of all of us physicists. An early example of unification is Sir Newton: he unified apples and planets. It was a revolutionary thought: the same law of physics applied to both terrestrial bodies, like an apple, and celestial bodies, like planets. Out of this unification came two important theories, Newton's law of mechanics, and the inverse-square law of gravity. A more familiar example is Maxwell, who unified 


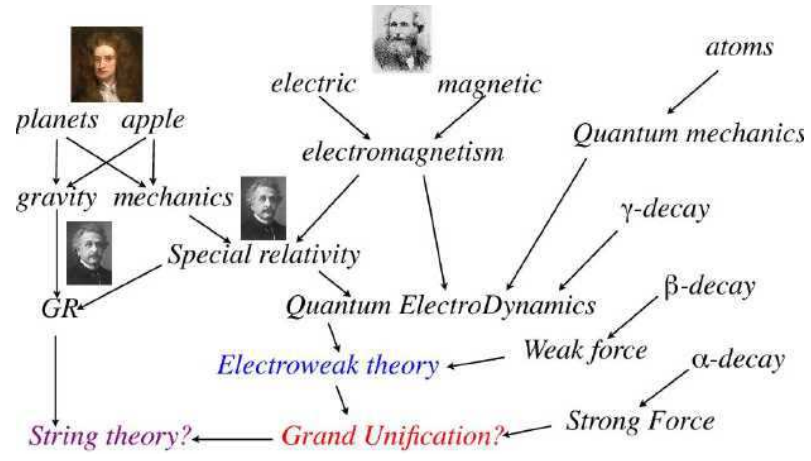

Figure 21. A brief history of unification in physics.

electric and magnetic forces. At the time of Einstein, there were also strange phenomena in atomic physics that led to quantum mechanics. In addition, there were even more mysterious phenomena in nuclear physics, such as $\alpha$-decay, $\beta$-decay, and $\gamma$-decay.

Later, there was an important unification in physics which somehow people don't talk about much. It is Quantum ElectroDynamics (QED), that unifies special relativity, electromagnetism, quantum mechanics, and some other phenomena such as nuclear $\gamma$-decay. It is an incredibly successful theory that predicts the magnetic moment of the electron down to its twelfth digit. It is equally incredible that experiments can measure it down to its twelfth digit, and all twelve digits agree with each other. This is a great triumph for the general idea of unification in physics.

The other phenomena led to discoveries of new forces. The nuclear $\beta$-decay was the first manifestation of the weak force, while the $\alpha$-decay was that of the strong force. We are now just about to achieve the next layer of unification, between QED and the weak force. Beyond that, we are still at the stage of speculation. The strong force may be further unified with the electroweak forces into a single force; it is called the grand unification. We also would like to see gravity unified with the other forces. Currently the best bet is string theory.

We are indeed just about to achieve the next layer of unification. Figure 22 shows the strengths of electromagnetic and weak forces as a function of the energy scale. The first manifestation of the weak force, nuclear $\beta$-decay, was measured at much lower energies, off the scale in Fig. 22] where the strengths of the two forces were many orders of magnitude dif-

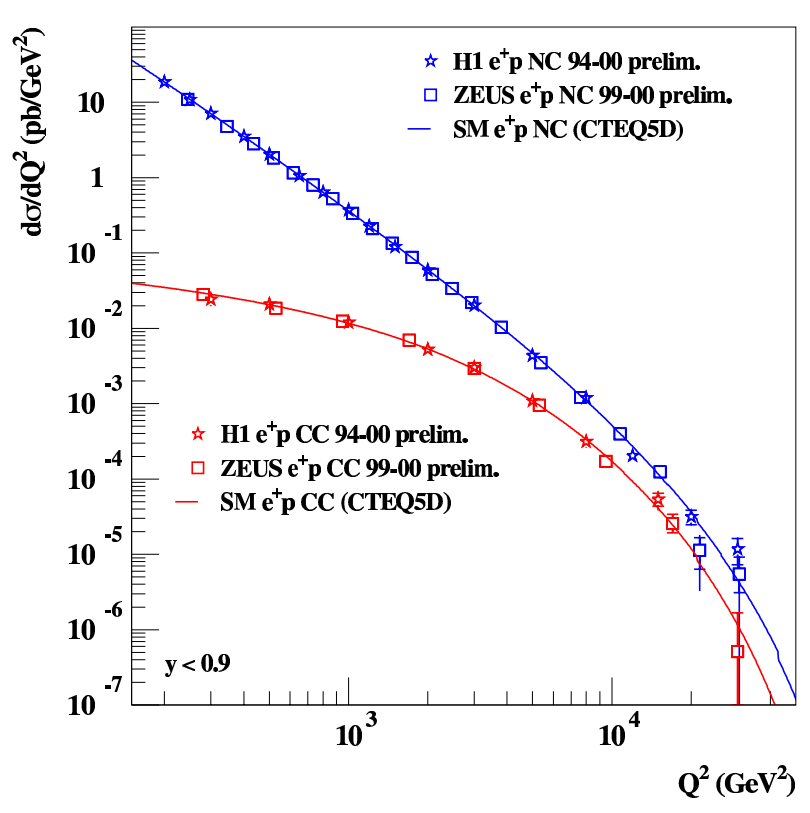

Figure 22. We are heading towards unification of the electromagnetism and the weak force 17

ferent. However our predecessors figured out that they are supposed to be of the same kind; an amazing insight. After many decades we inched up in energy, and are finally approaching the energy scale where they indeed become the same. It has been a long-term goal since the 1960's and we are getting there! However the important missing link is the Higgs boson as we talked about already.

Beyond the unification of electromagnetism and the weak force at the $\mathrm{TeV}$-scale, how do we gain any information about the next layer, the grand unification? We have all seen during the Symposium that the strengths (gauge couplings) of the three forces, $S U(3), S U(2)$, and $U(1)$, appear to become equal at a very high-energy scale $10^{16} \mathrm{GeV}$, if the Standard Model is Supersymmetric. The energy scale appears so remote that we may not gain any further information. However, if Supersymmetry is discovered, and the masses of the new particles are measured to a high precision by combining the data from the LHC and the LC, we will have a quantitative test of grand unification. The superpartners of the gauge bosons, gauginos, should have masses that unify at the same energy scale where the gauge couplings unify. This is a highly non-trivial test of whether forces unify. If this happens, we would definitely want to see proton decay! This is a wonderful example of how, once the hierarchy problem is solved, the solution itself will 


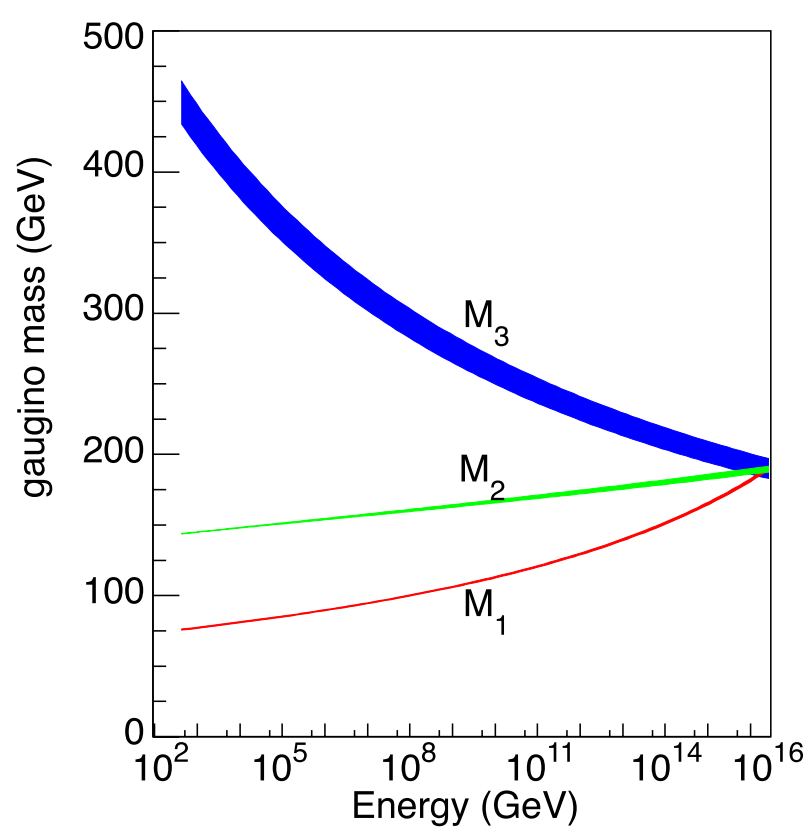

Figure 23. If Supersymmetry is found, it will provide a further probe to shorter distance scale physics, such as testing grand unification using the gaugino masses 18

provide new probes to physics that directly address the big questions.

Once the hierarchy problem is solved, we can systematically look for effects from physics at high energies. They can be parameterized as effective operators added to the Standard Model,

$$
\mathcal{L}=\mathcal{L}_{\mathrm{SM}}+\frac{1}{\Lambda} \mathcal{L}_{5}+\frac{1}{\Lambda^{2}} \mathcal{L}_{6}+\cdots
$$

where $\Lambda$ is the high energy scale of new physics. The effects in $\mathcal{L}_{5}$ are suppressed by a single power of the high energy scale, $\mathcal{L}_{6}$ by two powers, etc.. What terms there can be have been classified systematically by Weinberg, and there are many terms suppressed by two powers:

$$
\begin{gathered}
\mathcal{L}_{6} \supset Q Q Q L, \bar{L} \sigma^{\mu \nu} W_{\mu \nu} H e, W_{\nu}^{\mu} W_{\lambda}^{\nu} B_{\mu}^{\lambda}, \\
\bar{s} d \bar{s} d,\left(H^{\dagger} D_{\mu} H\right)\left(H^{\dagger} D^{\mu} H\right), \cdots .
\end{gathered}
$$

The examples here contribute to proton decay, $g-2$, the anomalous triple gauge boson vertex, $K^{0}-\bar{K}^{0}$ mixing, and the $\rho$-parameter, respectively. It is interesting that there is only one operator suppressed by a single power:

$$
\mathcal{L}_{5}=(L H)(L H)
$$

After substituting the expectation value of the Higgs,

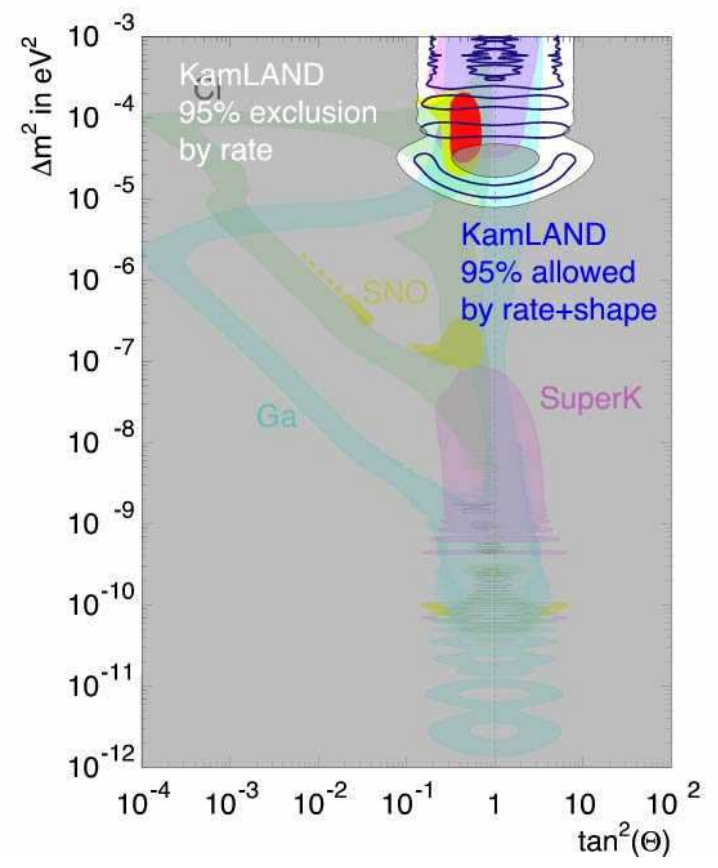

Figure 24. Solar neutrino data and reactor data converged on the Large Mixing Angle solution.24

the Lagrangian becomes

$$
\mathcal{L}=\frac{1}{\Lambda}(L H)(L H) \rightarrow \frac{1}{\Lambda}(L\langle H\rangle)(L\langle H\rangle)=m_{\nu} \nu \nu,
$$

nothing but the neutrino mass.

Therefore the neutrino mass plays a very unique role. It is the lowest-order effect of physics at short distances. This is a very tiny effect. Any kinematical effects of the neutrino mass are suppressed by $\left(m_{\nu} / E_{\nu}\right)^{2}$, and for $m_{\nu} \sim 1 \mathrm{eV}$ which we now know is already too large and $E_{\nu} \sim 1 \mathrm{GeV}$ for typical accelerator-based neutrino experiments, it is as small as $\left(m_{\nu} / E_{\nu}\right)^{2} \sim 10^{-18}$. At first sight, there is no hope to probe such a small number. However, any physicist knows that interferometry is a sensitive method to probe extremely tiny effects. For interferometry to work, we need a coherent source. Fortunately there are many coherent sources of neutrinos in Nature: the Sun, cosmic rays, reactors (not quite Nature), etc.. We also need interference for an interferometer to work. Fortunately, there are large mixing angles that make the interference possible. We also need long baselines to enhance the tiny effects. Again fortunately there are many long baselines available, 
such as the size of the Sun, the size of the Earth, etc.. Nature was very kind to provide all the necessary conditions for interferometry to us! Neutrino interferometry, a.k.a. neutrino oscillation, is a unique tool to study physics at very high energy scales. Indeed, the naïve interpretation of the neutrino oscillation results we heard about during this conference suggests $\Lambda \sim 10^{15} \mathrm{GeV}$ ! This gives an important look at the physics of grand unification.

\section{Horizontal}

The Horizontal Questions are about the flavor. As we witnessed during this conference, this is a historic era in flavor physics. In the lepton sector, Cowan and Reines detected neutrinos from a nuclear power reactor back in 1956, but we hadn't learned much about the nature of neutrinos for decades. In 1998, SuperKamiokande announced the discovery of oscillation in atmospheric neutrinos ${ }^{19}$ In 2002, SNO established the flavor conversion in solar neutrinos 20 Later the same year, KamLAND decided the solution to the solar neutrino problem (Fig. 24) 21

In the quark sector, the progress is similarly spectacular. Back in 1964, Fitch and Cronin discovered indirect CP-violation in $K^{0}-\bar{K}^{0}$ mixing. Again we didn't learn much beyond it for decades. Then in 1998, CPLEAR established $T$-violation in the same system.22] In 1999, KTeV and NA48 established the direct CP-violation, $\varepsilon^{\prime} / \varepsilon^{23}$ In 2001, BaBar and Belle established indirect $\mathrm{CP}$-violation in the $B_{d}$ system, the first CP-violation in a system other than kaons 25 These results combined to confirm the KobayashiMaskawa theory of CP-violation (Fig. 25).

But there are even more questions to be answered. The main question is this: What distinguishes different generations? Three generation of particles have the same quantum numbers, but they look very different. They have masses hierarchically different by many orders of magnitude. They mix rather little. Both hierarchical masses and small mixings go against our "common sense" in quantum mechanics. If two states share exactly the same set of quantum numbers, you expect them to have similar energy levels, and you expect them to mix a lot. The lack of both suggests that there is an ordered structure behind the flavor.

Many theorists including myself think that there are probably hidden flavor quantum numbers that

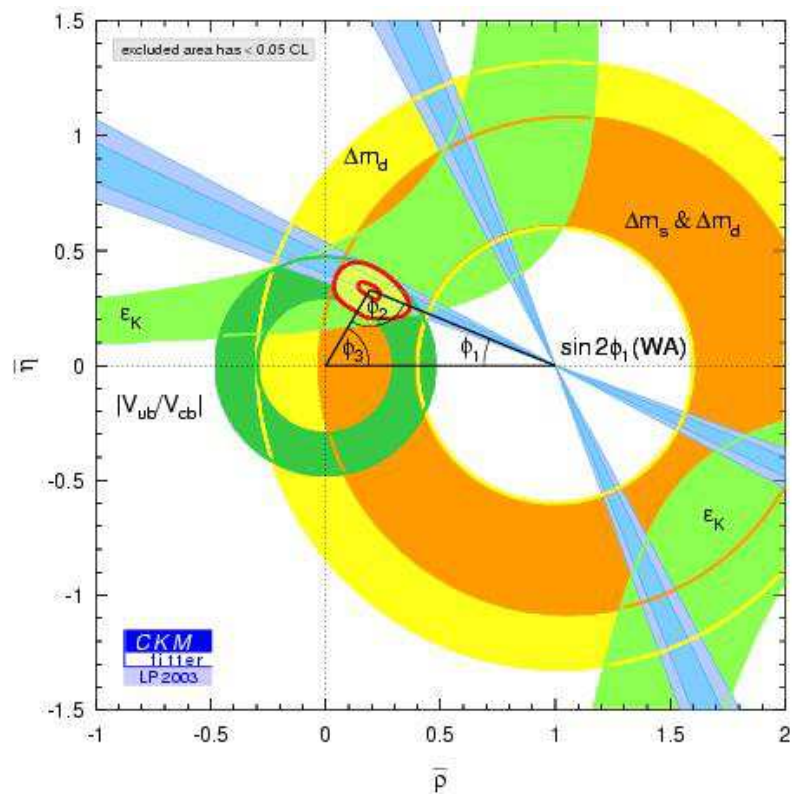

Figure 25. Consistency of various CKM parameter measurements shows the success of Kobayashi-Maskawa theory [26]

distinguish different generations. A quantum number means a new symmetry according to Noether's theorem: a flavor symmetry. This new symmetry must allow the top quark Yukawa coupling because it is $O(1)$. On the other hand, it forbids all the other Yukawa couplings so that all other quarks start out massless. But this symmetry must be only approximate, and is broken a little. This small symmetry breaking allows other Yukawa couplings, generating small and hierarchical Yukawas. Because different generations have different quantum numbers, they are not allowed to mix. Again the small symmetry breaking allows them to mix by small amounts.

Here is a toy model of a simple $U(1)$ flavor symmetry 27 basically introduce a new charge to all particles. This symmetry is broken by a small parameter $\langle\epsilon\rangle \sim 0.02$ of charge -1 . Let me assign charges to quarks and leptons in the following way,

$$
\begin{array}{r}
\mathbf{1 0}\left(Q, u_{R}, e_{R}\right)(+2,+1,0) \\
\mathbf{5}^{*}\left(L, d_{R}\right)(+1,+1,+1) .
\end{array}
$$

Here, I used grand-unified terminology of decuplet and quintet, and the three charges refer to the first, second, and third generation, respectively. This charge assignment keeps the top quarks, both left- and right-handed, neutral, and the top quark Yukawa coupling is allowed, while all other entries of 
the Yukawa matrices are forbidden. However, using $\epsilon$, we can fill in other entries as well. We find

$$
\begin{gathered}
M_{u} \sim\left(\begin{array}{lll}
\epsilon^{4} & \epsilon^{3} & \epsilon^{2} \\
\epsilon^{3} & \epsilon^{2} & \epsilon \\
\epsilon^{2} & \epsilon & 1
\end{array}\right), \\
M_{d} \sim\left(\begin{array}{lll}
\epsilon^{3} & \epsilon^{3} & \epsilon^{3} \\
\epsilon^{2} & \epsilon^{2} & \epsilon^{2} \\
\epsilon & \epsilon & \epsilon
\end{array}\right), \\
M_{l} \sim\left(\begin{array}{lll}
\epsilon^{3} & \epsilon^{2} & \epsilon \\
\epsilon^{3} & \epsilon^{2} & \epsilon \\
\epsilon^{3} & \epsilon^{2} & \epsilon
\end{array}\right) .
\end{gathered}
$$

It is easy to find the hierarchical mass eigenvalues,

$$
\begin{aligned}
& m_{u}: m_{c}: m_{t} \sim m_{d}^{2}: m_{s}^{2}: m_{b}^{2} \\
& \sim m_{e}^{2}: m_{\mu}^{2}: m_{\tau}^{2} \sim \epsilon^{4}: \epsilon^{2}: 1,
\end{aligned}
$$

which works pretty well especially given how simple the toy model is. The mixing angles are also suppressed by powers in $\epsilon$, and they all work out within a factor of 5 or so.

It is exciting that new data from neutrinos are already providing significant new information about flavor symmetries. As you know, neutrino data has been full of surprises. All mixing angles are large, except for $U_{e 3}$ which has not been measured. In particular, the atmospheric neutrino mixing appears maximal. Two mass-squared splittings are not very different, $\Delta m_{\text {solar }}^{2} / \Delta m_{\text {atmospheric }}^{2} \sim 1 / 30$. The hierarchy in masses rather than (masses) ${ }^{2}$ is the square root of this, $\sqrt{1 / 30}=0.2$. This isn't much of a hierarchy. Now we can ask the question of whether there is a symmetry or structure behind the neutrinos.

As far as we can tell, we don't need any symmetry or structure behind neutrinos, unlike the quark and charged leptons. If you run a Monte Carlo of random complex three-by-three matrices with the seesaw mechanism, you find that the maximal mixing is the most likely outcome if plotted against $\sin ^{2} 2 \theta$, and the peak in $\Delta m_{\text {solar }}^{2} / \Delta m_{\text {atmospheric }}^{2}$ is about $1 / 10$. Apparently no particular structure in the neutrino mass matrix is needed. I called this observation "anarchy" in neutrinos. Actually, the charge assignments I discussed earlier did not distinguish the three generation of neutrinos at all; and we do expect anarchy that is consistent with the current data.

Of course there are other proposals to understand the neutrino masses and mixings together with quarks and charged leptons. Table 2 shows a list of
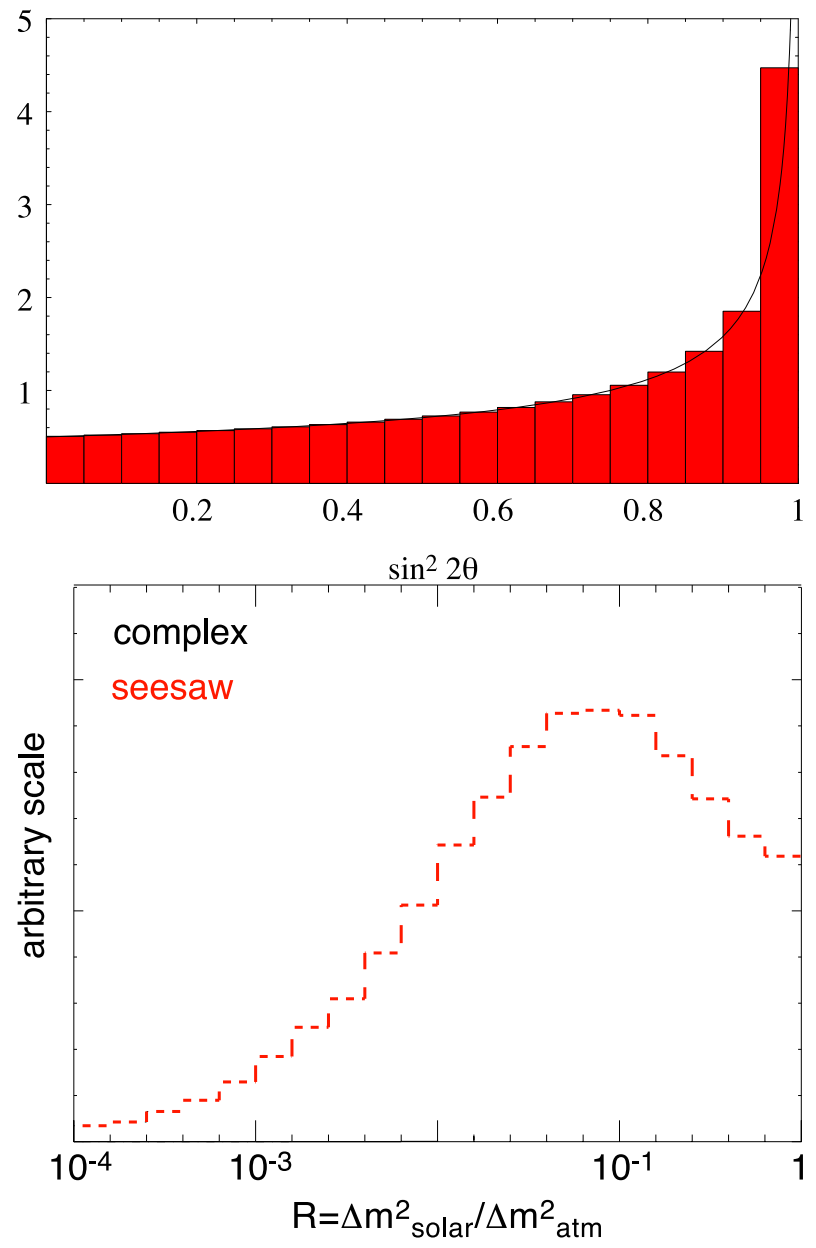

Figure 26. Random three-by-three matrices show distributions quite consistent with the observed patterns of neutrino masses and mixings 27 Top: $\sin ^{2} 2 \theta_{23}$. Bottom: $\Delta m_{\text {solar }}^{2} / \Delta m_{\text {atmospheric }}^{2}$.

proposed models of flavor symmetries as of October 2002. By December, KamLAND excluded the third and fourth flavor symmetries because they predicted other solutions to the solar neutrino problem. The survivors will be put to further tests soon. They predict different orders of magnitude for $\theta_{13}, O(1), O(\lambda)$, or $O\left(\lambda^{2}\right)$. If a more precise measurement of $\theta_{13}$ turns out to give $\sin ^{2} 2 \theta_{23}=1.00 \pm 0.01$, we would probably want a reason why it is so maximal, implying a new symmetry in the neutrino sector.

We'd like to push this program further to narrow down the choice of flavor symmetries. We basically need more and more flavor parameters. In fact, any $\mathrm{TeV}$-scale physics would have a new flavor structure that affects flavor physics significantly. Let me take Supersymmetry as an example. Squarks and slep- 


\begin{tabular}{|c|c|c|c|c|c|c|}
\hline Model & parameters & $d_{23}$ & $\Delta m_{12}^{2} /\left|\Delta m_{23}^{2}\right|$ & $U_{e 3}$ & $\tan ^{2} \theta_{12}$ & $\tan ^{2} \theta_{23}$ \\
\hline $\mathrm{A}$ & $b=0$ & $\mathrm{O}(1)$ & $\mathrm{O}(1)$ & $\mathrm{O}(1)$ & $\mathrm{O}(1)$ & $\mathrm{O}(1)$ \\
\hline $\mathrm{SA}$ & $b=1$ & $\mathrm{O}(1)$ & $\mathrm{O}\left(d_{23}^{2}\right)$ & $\mathrm{O}(\lambda)$ & $\mathrm{O}\left(\lambda^{2} / d_{23}^{2}\right)$ & $\mathrm{O}(1)$ \\
\hline $\mathrm{H}_{\mathrm{II}}$ & $a=1, b=2$ & $\mathrm{O}\left(\lambda^{2}\right)$ & $\mathrm{O}\left(\lambda^{4}\right)$ & $\mathrm{O}\left(\lambda^{2}\right)$ & $\mathrm{O}(1)$ & $\mathrm{O}(1)$ \\
\hline $\mathrm{H}_{\mathrm{I}}$ & $a=1, b=2$ & 0 & $\mathrm{O}\left(\lambda^{6}\right)$ & $\mathrm{O}\left(\lambda^{2}\right)$ & $\mathrm{O}(1)$ & $\mathrm{O}(1)$ \\
\hline $\mathrm{IH}$ & & $\mathrm{O}\left(\lambda^{4}\right)$ & $\mathrm{O}\left(\lambda^{2}\right)$ & $\mathrm{O}\left(\lambda^{2}\right)$ & $1+\mathrm{O}\left(\lambda^{2}\right)$ & $\mathrm{O}(1)$ \\
\hline
\end{tabular}

Table 2. Compilation of different flavor symmetry models and their predictions for neutrino masses and mixings as of Oct. $2002{ }^{28}$ The third and fourth rows were excluded by Dec. 2002. The next benchmark is $U_{e 3}$.

$$
\left(\begin{array}{c}
\tilde{s}_{R} \\
\tilde{s}_{R} \\
\tilde{s}_{R} \\
\tilde{\nu}_{\mu} \\
\tilde{\mu}
\end{array}\right) \leftrightarrow\left(\begin{array}{c}
\tilde{b}_{R} \\
\tilde{b}_{R} \\
\tilde{b}_{R} \\
\tilde{\nu}_{\tau} \\
\tilde{\tau}
\end{array}\right)
$$

Figure 27. The large $\nu_{\mu} \rightarrow \nu_{\tau}$ mixing suggests a large mixing of the whole $S U(5)$ multiplets and also of their superpartners.

tons come with their own mass matrices, in addition to quark and lepton mass matrices. Off-diagonal elements in squark/slepton mass matrices violate flavor. Therefore, a flavor symmetry that distinguishes different generations will automatically suppress the off-diagonal elements. If we can probe such small flavor violations in Supersymmetric loop diagrams, we would like to identify patterns in them, and eventually deduce the required symmetry behind them. Basically, we try to repeat what Gell-Mann and Okubo did in baryon and meson masses to identify the symmetry behind masses and mixings.

Different models differ in flavor quantum number assignments. Different quantum number assignments lead to different consequences for $\theta_{13}$, the matter effect, CP-violation, $B$-physics, $K$-physics, Lepton Flavor Violation, proton decay, and practically anything we can imagine that involves flavor. This way, we hope to identify the underlying flavor symmetry. I admit this is a long shot. We even don't know the energy scale of the physics of flavor. It may turn out to be too remote to access directly in experiments. But I'd like to argue that this is not necessarily bad. In archaeology, you don't reproduce the events in the laboratory. But once you have enough circumstantial evidence of fossils, artifacts, geological records, etc., that are consistent with a reasonable hypothesis, you eventually believe it. It may not be a formal proof at the level particle physicists are accustomed to, but it is nonetheless the next best thing. A good example is the cosmic microwave background. It is a wonderfully colorful, sexy fossil, and we can extract so much information out of it. We don't recreate the Big Bang, but we have already learned so much and we will learn even more from the CMB.

I'd like to emphasize that this program will be a collaboration of energy-frontier experiments and low-energy flavor experiments. We need to know the TeV-scale physics so that we know what runs inside the loops. We need to know their masses. Then the flavor data will allow us to extract flavor violations among the particles in the loops.

Here is one specific example we should pursue. ${ }^{29}$ We'd like to know if quarks and leptons have a common origin of flavor. As already mentioned, one striking discovery was that the $\nu_{\mu}$ and $\nu_{\tau} \operatorname{mix} a$ lot, maybe even maximally. Suppose you make it grand-unified. $s_{R}$ lives in the same multiplet as $\nu_{\mu}$, and $b_{R}$ with $\nu_{\tau}$. You'd expect a large mixing between $s_{R}$ and $b_{R}$, too (Fig. 27). But mixing among right-handed quarks completely drops out from the CKM phenomenology because there is no right-handed charged current (as far as we know). It looks like we can't probe this question. On the other hand, if there is Supersymmetry, a large mixing between $\tilde{s}_{R}$ and $\tilde{b}_{R}$ is physical, and can induce $O(1)$ effects in $b \rightarrow s$ transitions through loop diagrams (Fig. 28 top and center). Especially in leptogenesis that relies on $\mathrm{CP}$-violation in the neutrino sector, we expect CP-violation in $\tilde{s}_{R}-\tilde{b}_{R}$ mixing that may show up in $B$-physics.

For example, we may see CP-violation in $B_{s}$ mixing that can be studied in $B_{s} \rightarrow J / \psi+\phi$. The rates in $B_{d} \rightarrow X_{s} \ell^{+} \ell^{-}$may differ from the Standard Model and CP-violation may be seen. CPviolation in $B_{d} \rightarrow \phi+K_{S}$ may be different from that in $J / \psi+K_{S}$ within all the other constraints, such as $b \rightarrow s \gamma$ (Fig. 28 bottom). The current situation for $\phi K_{S}$ is somewhat confusing, with BaBar and Belle not consistent with each other. ${ }^{26}$ If they will settle 

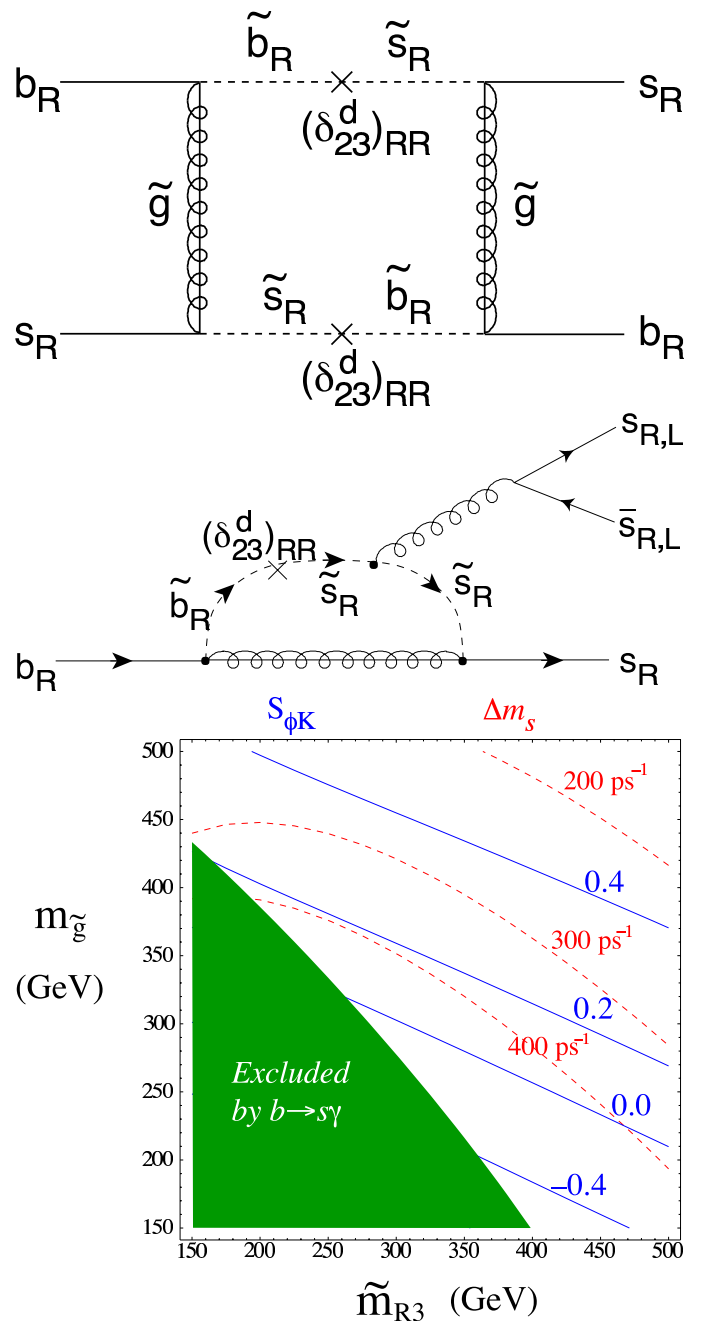

Figure 28. The impact of large $\tilde{s}_{R}{ }^{-} \tilde{b}_{R}$ mixing on $B$-physics. Top: possible contribution to the $B_{s}$ mixing. Center: possible contribution to the $B_{d} \rightarrow \phi K_{S}$ decay. Bottom: $S_{\phi K}$ in solid lines, $\Delta m_{s}$ in dotted lines, and the constraint from $b \rightarrow s \gamma$ in shaded region 30

in the middle, however, that may be the first indication of the common origin of flavor between quarks and leptons! I'm very much looking forward to more data.

After going through this program, suppose we identify a reasonable flavor symmetry that explains all data. Then we will be greedy enough to want to know what physics is behind the flavor symmetry. In the case of Gell-Mann-Okubo, once the $S U(3)$ flavor symmetry was identified, the next step was QCD. Clearly, we have to be very very lucky to get to that level. Nonetheless, it is useful to remember that the next level will crucially depend on what we find at
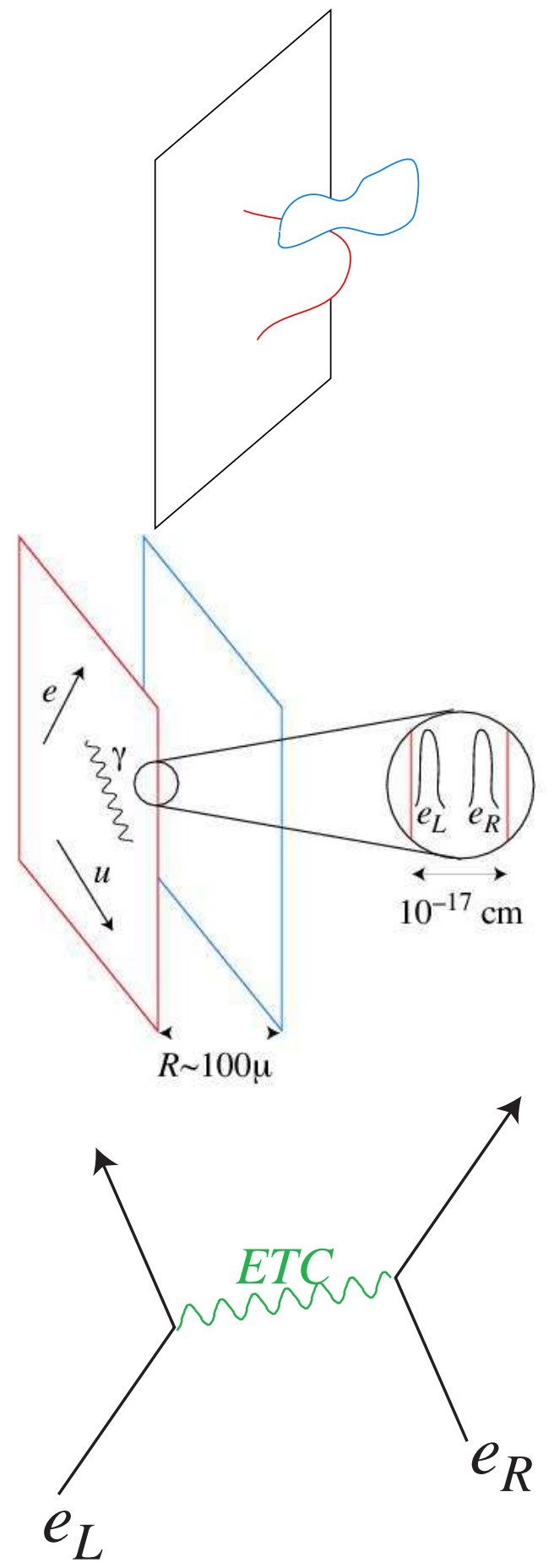

Figure 29. Different views on the origin of flavor symmetry depending on the outcome of the TeV-scale physics. Top: string origin in Supersymmetric models. Center: physical dislocation of different generations within a fat brane in models with hidden dimensions. Bottom: exchange of new massive gauge bosons at $100 \mathrm{TeV}$ scale in technicolor models. 
the $\mathrm{TeV}$-scale. For example, if the $\mathrm{TeV}$-scale turns out to be Supersymmetry, the flavor symmetry may be a consequence of the anomalous $U(1)$ gauge group with the Green-Schwarz mechanism from string theory (Fig. 29, top) $\stackrel{31}{\text { If }}$ it is hidden dimensions, it may be that the three-dimensional brane we live on is fat enough so that different generations are physically dislocated within the brane, providing an effective flavor symmetry (Fig. [29] center). $\frac{32}{6}$ If it is technicolor, it may be due to a new broken gauge interaction at the $100 \mathrm{TeV}$ scale (Fig. [29] bottom) 33 I certainly can't see far enough to know how things will play out.

\section{Conclusion}

What I'm looking forward to seeing in the next twenty years is a synergy of many different approaches in particle physics. The big questions I've listed at the beginning of my talk are all very ambitious questions. They are elusive. There is no guarantee that we can answer them.

But we know what the main obstacle is. It is the cloud of the $\mathrm{TeV}$-scale that is preventing us from obtaining clear views. And we are getting there. We have to make sure that there are many different approaches diverse enough to determine what is going on at the $\mathrm{TeV}$-scale. They will converge to reveal the big picture. Even though what I'm saying is ambitious, it is conceivable. And this idea of synergy applies to any scenario of $\mathrm{TeV}$-scale physics, as far as I can tell.

Given all this discussion, the outlook for the next twenty years is:

\section{Bright!}

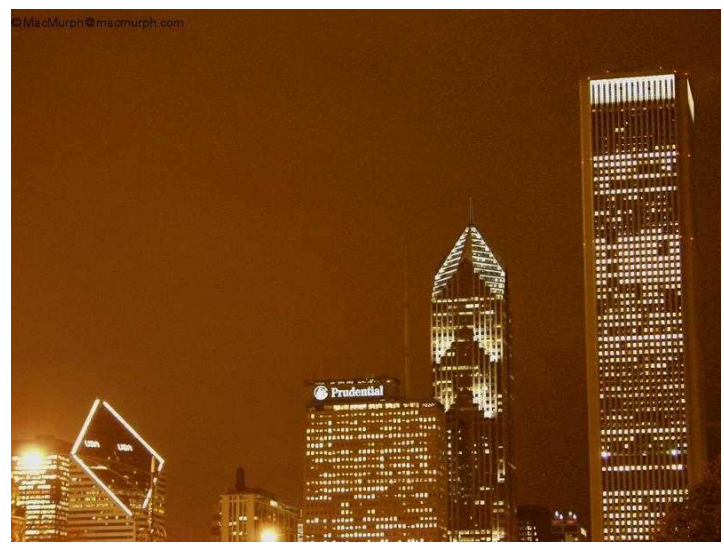

\section{References}

1. http://www-visualmedia.fnal.gov/VMS_Site /gallery/stillphotos/1995/95-759D.jpg

2. H. Murayama, "Supersymmetry," Talk given at 22nd INS International Symposium on Physics with High Energy Colliders, Tokyo, Japan, 8-10 Mar 1994. Published in Proceedings of INS Symposium, World Scientific, 1994. arXiv:hep-ph/9410285.

3. N. Arkani-Hamed, A. G. Cohen and H. Georgi, Phys. Lett. B 513, 232 (2001) [arXiv:hep-ph/0105239]; N. Arkani-Hamed, A. G. Cohen, T. Gregoire and J. G. Wacker, JHEP 0208, 020 (2002) [arXiv:hepph/0202089]; N. Arkani-Hamed, A. G. Cohen, E. Katz and A. E. Nelson, JHEP 0207, 034 (2002) [arXiv:hep-ph/0206021].

4. N. S. Manton, Nucl. Phys. B 158, 141 (1979). C. Csaki, C. Grojean and H. Murayama, Phys. Rev. D 67, 085012 (2003) [arXiv:hep-ph/0210133].

5. C. Csaki, C. Grojean, H. Murayama, L. Pilo and J. Terning, arXiv:hep-ph/0305237. C. Csaki, C. Grojean, L. Pilo and J. Terning, arXiv:hep-ph/0308038. Y. Nomura, arXiv:hep-ph/0309189.

6. H. Murayama, arXiv:hep-ph/0307293.

7. J. A. Aguilar-Saavedra et al. [ECFA/DESY LC Physics Working Group Collaboration], "TESLA Technical Design Report Part III: Physics at an e+eLinear Collider," arXiv:hep-ph/0106315.

8. H. Bachacou, I. Hinchliffe and F. E. Paige, Phys. Rev. D 62, 015009 (2000) [arXiv:hep-ph/9907518].

9. T. Tsukamoto, K. Fujii, H. Murayama, M. Yamaguchi and Y. Okada, Phys. Rev. D 51, 3153 (1995).

10. http://dmtools.berkeley.edu

11. E.W. Kolb, Michael S. Turner, The Early Universe. Redwood City, USA: Addison-Wesley (1990) 547 p. (Frontiers in physics, 69).

12. D. N. Spergel et al., Astrophys. J. Suppl. 148, 175 (2003) [arXiv:astro-ph/0302209]. See also Licia Verde in this proceedings.

13. C. Alcock et al. [MACHO Collaboration], THE ASTROPHYSICAL JOURNAL, 499:L9-L12 (1998), arXiv:astro-ph/9803082. ibid., The Astrophysical Journal, 550:L169-L172 (2001).

14. N. Arkani-Hamed, L. J. Hall, C. F. Kolda and H. Murayama, Phys. Rev. Lett. 85, 4434 (2000) [arXiv:astro-ph/0005111].

15. A. Friedland, H. Murayama and M. Perelstein, Phys. Rev. D 67, 043519 (2003) [arXiv:astro-ph/0205520].

16. http://snap.lbl.gov/target2.jpg

17. M. Erdmann, talk presented at XX International Symposium on Lepton and Photon Interactions at High Energies, 23rd-28th July 2001, Rome Italy. Published in the proceedings, J. Lee-Franzini, (ed.), F. Bossi, (ed.) (Frascati), P. Franzini, (ed.) Int. J. Mod. Phys. A 17, 2925-3549 (2002). 
18. H. U. Martyn and G. A. Blair, arXiv:hep$\mathrm{ph} / 9910416$.

19. Y. Fukuda et al. [Super-Kamiokande Collaboration], Phys. Rev. Lett. 81, 1562 (1998) [arXiv:hepex/9807003]. See also Koichiro Nishikawa in this proceedings.

20. Q. R. Ahmad et al. [SNO Collaboration], Phys. Rev. Lett. 89, 011301 (2002) [arXiv:nucl-ex/0204008]. See also Alain Bellerive in this proceedings.

21. K. Eguchi et al. [KamLAND Collaboration], Phys. Rev. Lett. 90, 021802 (2003) [arXiv:hepex/0212021]. See also Kunio Inoue in this proceedings.

22. A. Angelopoulos et al. [CPLEAR Collaboration], Phys. Lett. B 444, 43 (1998).

23. A. Alavi-Harati et al. [KTeV Collaboration], Phys. Rev. Lett. 83, 22 (1999) [arXiv:hep-ex/9905060]. V. Fanti et al. [NA48 Collaboration], Phys. Lett. B 465, 335 (1999) [arXiv:hep-ex/9909022].

24. http://hitoshi.berkeley.edu/neutrino

25. B. Aubert et al. [BABAR Collaboration], Phys. Rev.
Lett. 87, 091801 (2001) [arXiv:hep-ex/0107013]. K. Abe et al. [Belle Collaboration], Phys. Rev. Lett. 87, 091802 (2001) [arXiv:hep-ex/0107061].

26. Tom Browder in this proceedings.

27. N. Haba and H. Murayama, Phys. Rev. D 63, 053010 (2001) [arXiv:hep-ph/0009174].

28. G. Altarelli, F. Feruglio and I. Masina, JHEP 0301, 035 (2003) [arXiv:hep-ph/0210342].

29. D. Chang, A. Masiero and H. Murayama, Phys. Rev. D 67, 075013 (2003) [arXiv:hep-ph/0205111].

30. R. Harnik, D. T. Larson, H. Murayama and A. Pierce, arXiv:hep-ph/0212180.

31. L. E. Ibanez and G. G. Ross, Phys. Lett. B 332, 100 (1994) [arXiv:hep-ph/9403338]. P. Binetruy and P. Ramond, Phys. Lett. B 350, 49 (1995) [arXiv:hepph/9412385].

32. N. Arkani-Hamed and M. Schmaltz, Phys. Rev. D 61, 033005 (2000) [arXiv:hep-ph/9903417].

33. E. Eichten and K. D. Lane, Phys. Lett. B 90, 125 (1980). 


\section{DISCUSSION}

Bennie Ward (Baylor University \& University of Tennessee): In your discussion of the hierarchy problem you did not mention the anthropic principle. Could you please comment?

Hitoshi Murayama: As Ed said in the previous talk, I don't see the anthropic principle as the solution to a physical question. I suppose you can't exclude it, however.

John Collins (Penn State): You said that the Standard Model breaks down at a scale of around a TeV. How do you reconcile this with the fact the renormalized Standard Model is consistent to much higher energies?

Hitoshi Murayama: It is a matter of definition what you mean by "breaks down." The Standard Model is certainly consistent as a renormalizable field theory, and can be applied to arbitrary high energies in that sense. However, we view it as a low-energy effective field theory rather than the ultimate theory of everything, and therefore it has an ultraviolet cut-off. My definition of the Standard Model breakdown is the fact that the perturbative corrections exceed the bare Higgs mass-squared parameter as the cut-off is raised beyond $\mathrm{TeV}$. It is the same sense as when Landau and Lifshitz discussed the breakdown of classical electrodynamics at the classical radius of the electron. 